\title{
Origins of Elasticity in Intermediate Filament Networks
}

\section{Citation}

Lin, Yi-Chia, Norman Y. Yao, Chase P. Broedersz, Harald Herrmann, Fred C. MacKintosh, and David A. Weitz. 2010. "Origins of Elasticity in Intermediate Filament Networks." Physical Review Letters104 (5): 058101. https://doi.org/10.1103/PhysRevLett.104.058101.

\section{Permanent link}

http://nrs.harvard.edu/urn-3:HUL.InstRepos:41511267

\section{Terms of Use}

This article was downloaded from Harvard University's DASH repository, and is made available under the terms and conditions applicable to Other Posted Material, as set forth at http:// nrs.harvard.edu/urn-3:HUL.InstRepos:dash.current.terms-of-use\#LAA

\section{Share Your Story}

The Harvard community has made this article openly available.

Please share how this access benefits you. Submit a story.

Accessibility 


\title{
Origins of Elasticity in Intermediate Filament Networks
}

\author{
Yi-Chia Lin, ${ }^{1}$ Norman Y. Yao, ${ }^{1}$ Chase P. Broedersz, ${ }^{2}$ Harald Herrmann, ${ }^{3}$ Fred C. MacKintosh, ${ }^{2}$ and David A. Weitz ${ }^{1,4}$ \\ ${ }^{1}$ Department of Physics, Harvard University, Cambridge, Massachusetts 02138, USA \\ ${ }^{2}$ Department of Physics and Astronomy, Vrije Universiteit, Amsterdam, The Netherlands \\ ${ }^{3}$ Department of Cell Biology, German Cancer Research Center, Heidelberg, Germany \\ ${ }^{4}$ School of Engineering and Applied Sciences, Harvard University, Cambridge, Massachusetts 02138, USA \\ (Received 7 August 2009; revised manuscript received 1 December 2009; published 1 February 2010)
}

\begin{abstract}
Intermediate filaments are common structural elements found in abundance in all metazoan cells, where they form networks that contribute to the elasticity. Here, we report measurements of the linear and nonlinear viscoelasticity of networks of two distinct intermediate filaments, vimentin and neurofilaments. Both exhibit predominantly elastic behavior with strong nonlinear strain stiffening. We demonstrate that divalent ions behave as effective cross-linkers for both networks, and that the elasticity of these networks is consistent with the theory for that of semiflexible polymers.
\end{abstract}

DOI: 10.1103/PhysRevLett.104.058101

The mechanical response of cells depends largely on the structure and elasticity of their cytoskeleton, consisting of a variety of biopolymer networks, including filamentous actin, microtubules, and intermediate filaments (IFs) [1]. While filamentous actin and microtubules have been extensively studied, much less is known about IFs, although some key parameters such as their persistence length have been measured. Compared to actin and microtubules, IFs are more varied and specialized. Their networks are nevertheless cytoskeletal components contributing to the elasticity of the cell: there are five families of IFs found in a variety of cell types, ranging from muscles to neurons. Intermediate filament networks exhibit pronounced nonlinear elasticity similar to that observed in actin networks that are cross-linked. However, there are myriad associated actin-binding proteins that lead to this cross-linking; by contrast, fewer cross-linking proteins have been identified for IFs. Thus, the origin of the nonlinear elasticity in IF networks has not been identified.

Here, we investigate the elasticity of two different IF networks, vimentin and neurofilaments (NFs). The networks exhibit remarkably similar mechanical properties: they are weak elastic solids even at the lowest frequencies probed and they exhibit strong nonlinear strain stiffening over several decades in stress. This behavior requires cross-linking of the network and we show that divalent ions act as effective cross-linkers. By comparing the linear and nonlinear macroscopic behavior, we extract microscopic network parameters. These observations suggest a general design principle for regulating the elasticity of intermediate filament networks even in the absence of specific cross-linking proteins.

To explore the generality of this behavior, we use NFs, found only in neurons, and vimentin, found in nearly all mesenchymal cells. The main difference between these IFs lies in the length of their negatively charged carboxy terminal sidearms; NF sidearms are much longer than those of vimentin. Furthermore, optimal assembly is ob-
PACS numbers: 87.15.La, 83.60.Df, 83.80.Rs, 87.16.Ka

tained at different ionic conditions, with NFs favoring a $p \mathrm{H}$ of 6.2, where vimentin does not assemble properly [2]. Neurofilaments are purified from bovine spinal cords [35]: fresh tissue is homogenized, then centrifuged, after which the crude NF pellet is purified overnight on a discontinuous sucrose gradient with $0.8 \mathrm{M}$ sucrose $(5.9 \mathrm{ml})$, $1.5 \mathrm{M}$ sucrose $(1.3 \mathrm{ml})$ and $2.0 \mathrm{M}$ sucrose $(1.0 \mathrm{ml})$. The purified NF is then dialyzed for 76 hours and $120 \mu 1$ aliquots are flash frozen in liquid nitrogen and stored at $-80^{\circ} \mathrm{C}$. Human vimentin protein is expressed in Escherichia coli and purified from inclusion bodies [6]. The protein is stored at $-80^{\circ} \mathrm{C}$ in $8 \mathrm{M}$ urea, $5 \mathrm{mM}$ Tris$\mathrm{HCl}(p \mathrm{H}$ 7.5), 1 mM DTT, 1 mM EDTA, 0.1 mM EGTA, and $10 \mathrm{mM}$ methyl ammonium chloride. Twenty-four hours before use, we renature the protein from $8 \mathrm{M}$ urea by stepwise dialysis ( $6 \mathrm{M}, 4 \mathrm{M}, 2 \mathrm{M})$ into a solution of $5 \mathrm{mM}$ Tris- $\mathrm{HCl}, p \mathrm{H}$ 8.4, $1 \mathrm{mM}$ EDTA, $0.1 \mathrm{mM}$ EGTA, and $1 \mathrm{mM}$ DTT. The protein concentration is determined using a Bradford assay with bovine serum albumin (BSA) as a standard.

The mechanical response of intermediate filament networks is measured with a stress-controlled rheometer using a $2^{\circ} 20 \mathrm{~mm}$ cone-plate geometry for NFs and a $40 \mathrm{~mm}$ plate-plate geometry for vimentin (HR Nano, Malvern Instruments). Before rheological testing, neurofilament samples are thawed on ice, after which, varying concentrations of $\mathrm{Mg}^{2+}$ are added. Vimentin polymerization is initiated by adding $\mathrm{Mg}^{2+}$ and $1 / 10$ of the final sample volume of $10 \mathrm{X}$ polymerization buffer $(0.2 \mathrm{M}$ Tris- $\mathrm{HCl}, p \mathrm{H}$ 7.0, containing $1.6 \mathrm{M} \mathrm{NaCl}$ ). The samples are quickly loaded onto the rheometer and polymerized between the rheometer plates for $1 \mathrm{~h}$ at $25^{\circ} \mathrm{C}$, using a solvent trap to prevent drying. We measure the linear viscoelastic moduli $G^{\prime}(\omega), G^{\prime \prime}(\omega)$. In addition, we use large amplitude oscillatory measurements to qualify the network's nonlinearity. To quantify the nonlinear behavior we utilize a differential measurement $[7,8]$. The system is held at a constant average (pre-)stress $\sigma$, while the differential response $d \gamma$ to a 
small additional oscillatory stress $d \sigma$ is measured to yield the nonlinear tangent modulus $K=d \sigma / d \gamma$.

We first probe the frequency dependence of the linear viscoelastic moduli. Consistent with other biopolymer networks, both $G^{\prime}, G^{\prime \prime}$ depend weakly on frequency (from $0.01-10 \mathrm{~Hz}$ ), with $G^{\prime}$ much larger than $G^{\prime \prime}$, as shown in Fig. 1(a). This suggests the existence of soft cross-linked gels. We characterize the linear elasticity by the plateau modulus, $G_{0}$, the value of $G^{\prime}(\omega)$ at $0.1 \mathrm{~Hz}$. Interestingly, for purely entangled networks of similar size and concentration, we would expect the moduli of the two IF networks to be comparable; the fact that NFs are about an order of magnitude stiffer can be accounted for by cross-linking. The networks also exhibit dramatic strain stiffening above a critical strain $\gamma_{c}$, as shown in Fig. 1(b).

For both IFs, the elasticity depends strongly on the polymer concentration and also on the concentration of $\mathrm{Mg}^{2+}$ added to the solution [9]. By analogy to prior studies of biopolymer networks $[8,10]$, we examine the dependence on IF concentration, $c_{\mathrm{IF}}$, at a fixed mole ratio, $R$ of $\mathrm{Mg}^{2+}$ to IF. For both vimentin and NFs, the linear elastic modulus increases with filament concentration in a way similar to cross-linked biopolymer networks, in that the modulus scales slightly stronger than quadratically with filament concentration, as shown in Fig. 2(a). This is consistent with a theoretical model for cross-linked semiflexible polymers, in which the elasticity is dominated by entropic filament stretching; in this affine thermal model, the filaments are considered to be entropic springs, leading to $G_{0} \sim c_{\mathrm{IF}}^{11 / 5}$ [11]. In addition, we also find that $G_{0}$ scales directly with $c_{\mathrm{Mg}}$, as shown in Fig. 2(b), demonstrating the role of $\mathrm{Mg}^{2+}$ in the network elasticity for both IFs.

To elucidate the role of $\mathrm{Mg}^{2+}$, we investigate the nonlinear elastic regime by probing the differential moduli [8]. Above a critical stress $\sigma_{c}$, both IF networks display pronounced nonlinear stiffening with applied stress, exhibiting an approximate power law of 3/2, as shown in Fig. 3. This is consistent with theoretical expectations and pre-

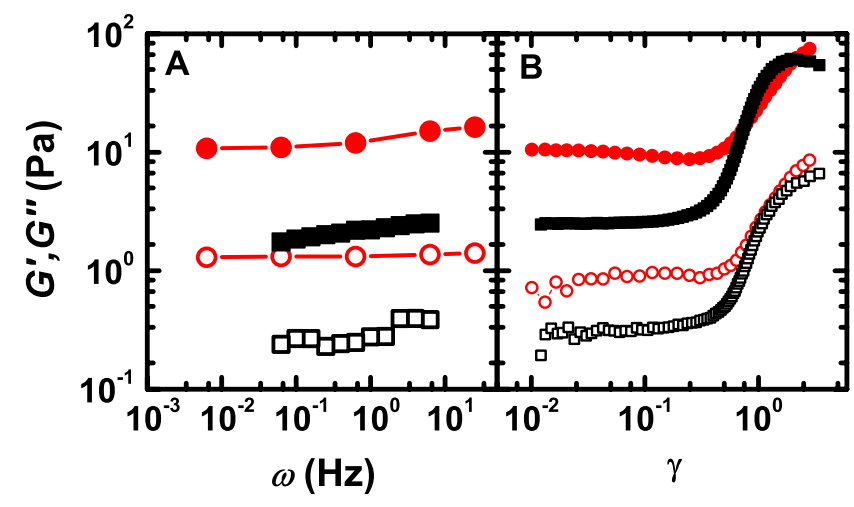

FIG. 1 (color online). (a) $G^{\prime}$ (solid symbols) and $G^{\prime \prime}$ (open symbols) as a function of frequency in the linear regime. (b) $G^{\prime}$ (solid symbols) and $G^{\prime \prime}$ (open symbols) as a function of strain, $\gamma$. Samples of vimentin (squares) and neurofilaments (circles) are probed at a $2 \mathrm{mg} / \mathrm{ml}$ filament concentration and a $5 \mathrm{mM} \mathrm{Mg}^{2+}$. vious experiments for cross-linked networks [8,10-12]. Interestingly, the predictions of the affine thermal model apply for networks cross-linked by molecular linkers and experiments have focused on actin-linkers such as scruin. The absence of molecular linkers in these IF networks suggests that ions may be playing the role of permanent effective molecular cross-links, lending insight into the nature of ionic interactions in IF networks. Previously, these ionic interactions have been understood within a framework of condensed counterions and salt-bridging on polyelectrolyte brushes [13-15]. We hypothesize that $\mathrm{Mg}^{2+}$ mediates attractive interactions between the negatively charged sidearms of the IFs thereby forming permanent cross-links analogous to molecular linkers. In particular, IF sidearms are likely collapsed flexible chains, and the cross-linking interactions would be mediated by a collection of divalent ions interwoven into the entangled sidearm structure. Although sidearms of a single chain will likely cross-link within themselves, only cross-linking between different chains will contribute to network elasticity. Since neurofilament sidearms are much longer than those of vimentin, their cross-linking may be more effective, accounting for the higher shear moduli and yield stresses observed for NF networks. Theoretically we expect a universal form for the nonlinear elastic response of the networks for all protein and ion concentrations. The data sets from both neurofilament and vimentin networks can, indeed, be scaled onto a single master curve, which is in good agreement with the theoretical prediction shown by the solid line in the inset of Fig. $3[8,10]$. Here, we fit each data set to the full theoretical curve, using two independent parameters: $\sigma$ is scaled by $\sigma_{c}$, while $K^{\prime}$ is scaled by the linear elastic modulus $G_{0}$. For both networks, we find excellent agreement with theory over approximately four decades in stress, although vimentin departs from the theoretical master curve at the highest stresses. This departure may be explained by considering the enthalpic contribution of filament backbone stretching [16], suggest-

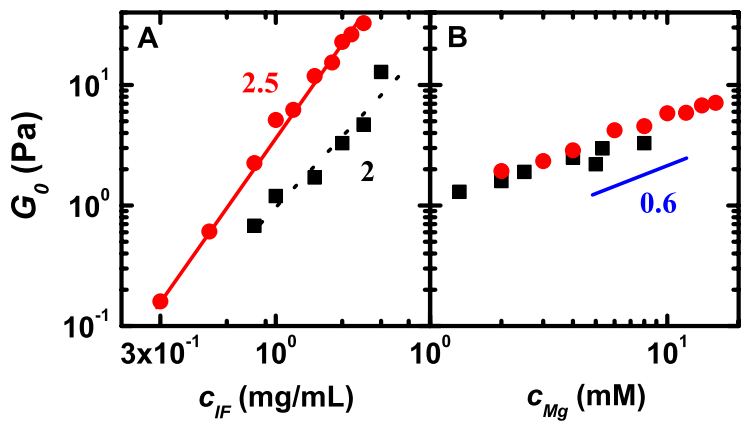

FIG. 2 (color online). (a) $G_{0}$ as a function of $c_{\mathrm{IF}}$ holding $R$ constant, where $R=1000$ for neurofilaments (circles) and $R=$ 215 for vimentin (squares). Solid line is obtained using a regression fit and depicts $G_{0} \sim c_{\mathrm{NF}}^{2.5}$ for neurofilaments, while dashed line depicts $G_{0} \sim c_{V}^{2}$. (b) $G_{0}$ as a function of $c_{\mathrm{Mg}}$ holding $c_{\mathrm{IF}}$ constant, where $c_{\mathrm{IF}}=1 \mathrm{mg} / \mathrm{ml}$ for neurofilaments (circles) and $c_{\mathrm{IF}}=2 \mathrm{mg} / \mathrm{ml}$ for vimentin (squares). 


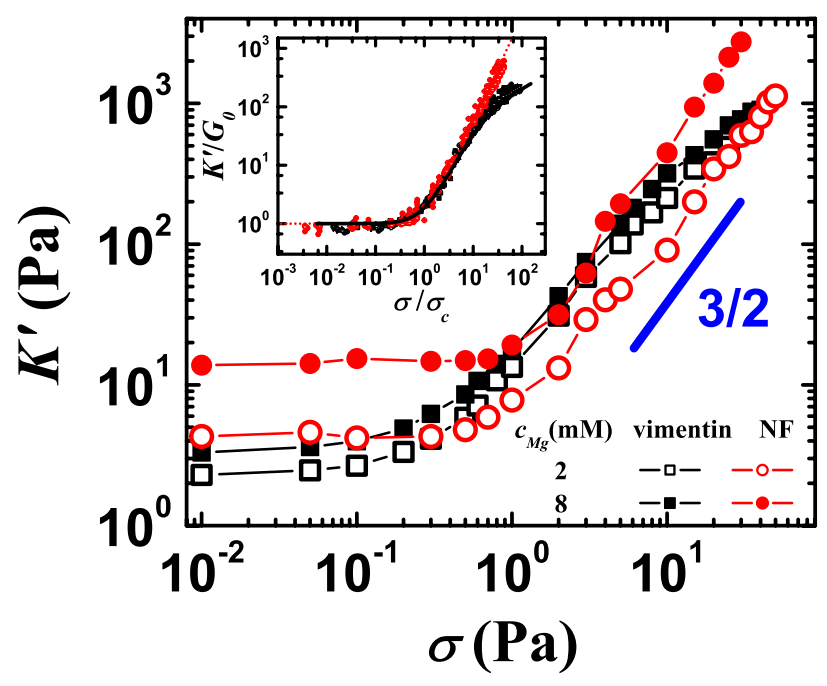

FIG. 3 (color online). $\quad K^{\prime}$ at $0.6 \mathrm{rad} / \mathrm{sec}$ as a function of $\sigma$ for vimentin and neurofilament networks: $\left[c_{\mathrm{Mg}}=2 \mathrm{mM}\right.$ (open symbols), $c_{\mathrm{Mg}}=8 \mathrm{mM}$ (solid symbols) and $c_{\mathrm{IF}}=2 \mathrm{mg} / \mathrm{ml}$ (all symbols)]. The solid line shows a $3 / 2$ power law. The inset shows the data sets rescaled by $\sigma_{c}$ and $G_{0}$ depicting the universal form of the stiffening response; the rescaled theory is indicated by the dashed red line, while the solid black line depicts the deviation due to enthalpic backbone stretching.

ing that the Young's modulus of vimentin is less than that of neurofilaments. In particular, utilizing an extensible wormlike chain model we are able to estimate vimentin's Young's modulus to be approximately $9 \mathrm{MPa}[17,18]$.

To further probe the mechanism of the network elasticity, we test the predicted relationship between $G_{0}$ and $\sigma_{c}$. Assuming an affine deformation, theory predicts the curve shown in the inset of Fig. 3, with

$$
G_{0}=6 \rho k_{B} T \frac{l_{p}^{2}}{l_{c}^{3}} \quad \text { and } \quad \sigma_{c}=\rho k_{B} T \frac{l_{p}}{l_{c}^{2}} .
$$

Here, $k_{B}$ is Boltzmann's constant, $T$ is the temperature, $\rho$ is the filament density in length per volume, $l_{p}$ is the persistence length, and $l_{c}$ is the average distance between crosslinks $[8,10,11,19]$. Since $\rho \propto c_{\mathrm{IF}}$, the model predicts that $c_{\mathrm{IF}}^{1 / 2} G_{0} \sim \sigma_{c}^{3 / 2}$, where the prefactor should depend only on $k_{B} T$ and $l_{p}$. In particular, this relationship is predicted to be independent of $l_{c}$, so that even data sets with different cross-link densities should collapse onto a single curve. Both vimentin and NF networks agree with this data collapse and scaling for a variety of different filament and $\mathrm{Mg}^{2+}$ concentrations, as shown in Fig. 4. In the case of NFs, the generality of this ionic cross-linking behavior is depicted by a qualitative collapse onto the same curve for other divalent ions such as $\mathrm{Ca}^{2+}$ and $\mathrm{Zn}^{2+}$. This scaling relates the linear elasticity of the networks to the stress at which nonlinear behavior begins as the filaments approach their full extension; therefore, it is independent of the scaling of $K^{\prime}$ with $\sigma$ in Fig. 3. This provides strong evidence that the linear elasticity of IF networks is also governed by entropic stretching.
The relative shift between the two curves in Fig. 4. can be explained by a difference in persistence lengths of the filaments. Remarkably, we can determine $l_{p}$ directly from the macroscopic network behavior under shear,

$$
l_{p}=\frac{1}{36} \rho k_{B} T \frac{G_{0}^{2}}{\sigma_{c}^{3}},
$$

where $\rho \approx 0.5 \times 10^{13} \mathrm{~m}^{-2}$ for both IF networks at a concentration of $4 \mu \mathrm{M}$. We find that for NFs, $l_{p} \approx 0.2 \mu \mathrm{m}$, while for vimentin, $l_{p} \approx 0.5 \mu \mathrm{m}$; both of these values are in good accord with previous experiments [20-23]. Furthermore, we also determine the cross-link density,

$$
l_{c}=6 l_{p} \frac{\sigma_{c}}{G_{0}},
$$

where we have obtained $l_{p}$ directly from bulk rheological parameters using Eq. (2). Direct determination of $l_{c}$ is difficult and has rarely been made in such systems [24]. Here, we find that $l_{c} \approx 0.3 \mu \mathrm{m}$ for NFs and $l_{c} \approx 0.6 \mu \mathrm{m}$ for vimentin. This is consistent with our hypothesis that the filaments are cross-linked on the scale $l_{p}$ and thus the networks can be characterized as semiflexible.

To further examine the behavior of $\mathrm{Mg}^{2+}$ as a crosslinker, we consider the scaling of $l_{c}$ as a function of both $R$ and $c_{\mathrm{IF}}$ [Eq. (3)]. Cross-linking occurs on the scale of the entanglement length $l_{e}$, which scales as $c_{\mathrm{IF}}^{-2 / 5}[11,18]$. Since our data suggest that $\mathrm{Mg}^{2+}$ is effectively a crosslinker, we expect $l_{c}$ to scale with $R^{-x}$ for some exponent $x$ $[8,10]$. Indeed, the data exhibit an approximate scaling, $l_{c} \sim R^{-x} c_{\mathrm{IF}}^{-y}=c_{\mathrm{Mg}}^{-x} c_{\mathrm{IF}}^{-y+x} ; x$ is approximately 0.23 for both IFs, measured at fixed $c_{\mathrm{IF}}$, and $y \approx 0.4$ for vimentin and $y \approx 0.5$ for neurofilaments, measured at fixed $R$, as shown in Fig. 5. Interestingly, the larger value of $y$ for NFs is consistent with the correspondingly stronger concentration

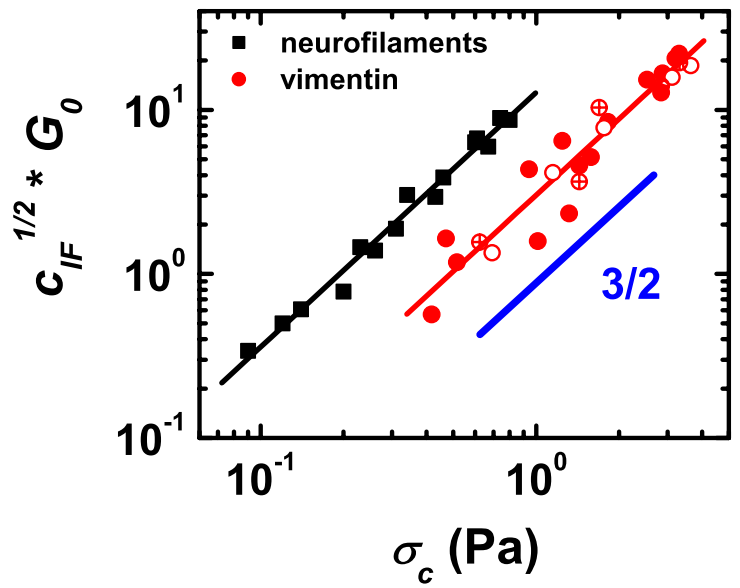

FIG. 4 (color online). The dependence of $c_{\mathrm{IF}}^{1 / 2} G_{0}$ on $\sigma_{c}$. Vimentin data points are obtained with $\mathrm{Mg}^{2+}$, while $\mathrm{NF}$ data points are obtained with $\mathrm{Mg}^{2+}$ (closed circles), $\mathrm{Ca}^{2+}$ (open circles), and $\mathrm{Zn}^{2+}$ (crossed circles). The solid lines reflect regression fits. The affine thermal model predicts a power law of $3 / 2$, shown for reference. 


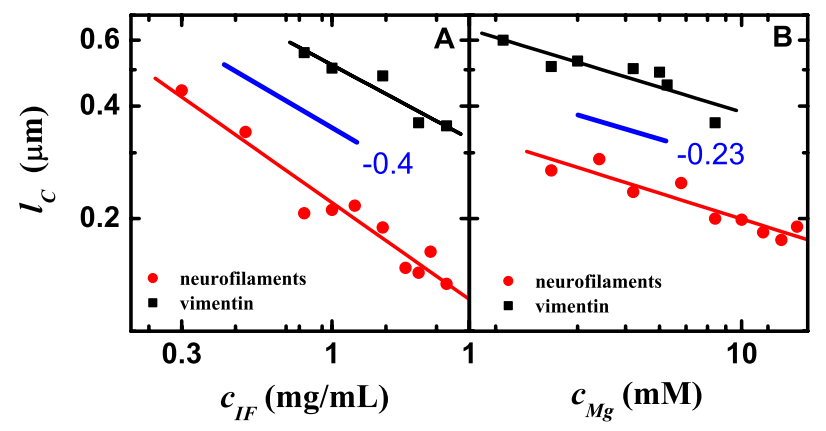

FIG. 5 (color online). (a) The dependence of $l_{c}$ on $c_{\mathrm{IF}}$ at fixed $R$, where $R=1000$ for NFs (circles) and $R=215$ for vimentin (squares). A power law of -0.4 is shown for reference. (b) The dependence of $l_{c}$ on $c_{\mathrm{Mg}}$, holding $c_{\mathrm{IF}}$ constant. A regression fit results in a power law of -0.23 for both vimentin and neurofilament networks.

dependence of $G_{0}$ (Fig. 2). This may be a consequence of denser cross-linking; then we expect $G_{0} \sim c_{\mathrm{NF}}^{5 / 2}$ [11]. Furthermore, as a consistency check for the value of $x$, we can also determine it directly from the $c_{\mathrm{Mg}}$ dependence of $G_{0}$. Inserting a scaling of $l_{c} \sim R^{-x}$ into Eq. (1) gives $G_{0} \sim c_{\mathrm{Mg}}^{3 x}$, holding $c_{\mathrm{IF}}$ constant. Based on the scaling of $G_{0}$ in Fig. 2(b), we find $x \approx 0.20$, in good accord with the value measured directly from the $c_{\mathrm{Mg}}$ dependence of $l_{c}$. Both the excellent agreement with theory and the internal consistency of our measurements provides convincing evidence that $\mathrm{Mg}^{2+}$ effectively cross-links IF networks. Moreover, we find similar behavior for $\mathrm{Ca}^{2+}$ and $\mathrm{Zn}^{2+}$.

The values we observe for $G_{0}$ and the maximum stress are consistent with most previous experiments [1,5], although one experiment with NFs yielded a smaller modulus and solution like behavior [25]. Remarkably, we can extract the microstructural parameters $l_{p}$ and $l_{c}$ directly from bulk rheology; values of $l_{p}$ are consistent with previous measurements [20], while values of $l_{c}$ are comparable to the expected mesh size $\xi \approx 1 / \sqrt{\rho}$. Compared with vimentin, NFs consistently exhibit smaller values of $l_{c}$, suggesting a more densely cross-linked network; this may result from the longer NF sidearms and concomitantly stronger electrostatic interactions. Measurements based on probe particle motion in IF networks have suggested larger values of $\xi$ [25]; however, recent measurements yield results which are more consistent with the expected value [26]. In addition, results from direct imaging clearly show $\xi$ of order a few hundred nanometers.

Vimentin and NF networks show striking similarity in their linear and nonlinear elastic behavior, both of which are governed by cross-linking due to divalent ions. Intriguingly, divalent ions play a role nearly identical to that of molecular cross-linkers in F-actin networks. This suggests that there is a strong affinity of the divalent ions to the IFs; thus, a large fraction of the ions must be bound hence becoming effective molecular cross-links. Despite the similarities in their mechanical behavior, the nonlinear rheology of vimentin shows a clear departure from that of NFs at the highest stresses; this results from the enthalpic contribution of filament backbone stretching [16]. By contrast NFs do not exhibit a measurable filament compliance, implying that their Young's modulus is larger than that of vimentin; this is surprising given the similar molecular architecture of the backbone of the two IFs. Interestingly, this may reflect another important role of the sidearms: they might also affect the enthalpic stretching of filaments. However, this conclusion is based on macroscopic rheology, and direct force-extension measurements of individual filament stretching are needed to confirm this. Such filament extension experiments may also elucidate the nature of the electrostatic interactions that mediate effective molecular cross-linking between IFs.

We acknowledge the insights of Fyl Pincus and discussions with P. Janmey on neurofilament rheology. This work was supported by the NSF (DMR-0602684 and CTS0505929), the Harvard MRSEC (DMR-0213805), and by FOM/NWO. N. Yao acknowledges support from the DOE (FG02-97ER25308) and H. Herrmann from the DFG (HE1853 and BA 2186/2-1).

[1] O. I. Wagner et al., Exp. Cell Res. 313, 2228 (2007).

[2] H. Herrmann and U. Aebi, Guidebook to the Cytoskeletal and Motor Proteins (Oxford University Press, Oxford, 1999).

[3] A. Delacourte et al., Biochem. J. 191, 543 (1980).

[4] J. F. Leterrier and J. Eyer, Biochem. J. 245, 93 (1987).

[5] J. F. Leterrier et al., J. Biol. Chem. 271, 15687 (1996).

[6] H. Herrmann, I. Hofmann, and W. W. Franke, J. Mol. Biol. 223, 637 (1992).

[7] N. Y. Yao, R. Larsen, and D. A. Weitz, J. Rheol. 52, 1013 (2008).

[8] M. L. Gardel et al., Science 304, 1301 (2004).

[9] J. Kas et al., Biophys. J. 70, 609 (1996).

[10] M. L. Gardel et al., Phys. Rev. Lett. 93, 188102 (2004).

[11] F. C. MacKintosh, J. Kas, and P. A. Janmey, Phys. Rev. Lett. 75, 4425 (1995).

[12] M. Fixman and J. Kovac, J. Chem. Phys. 58, 4 (1973).

[13] E. B. Zhulina and F. A. M. Leermakers, Biophys. J. 93, 1421 (2007).

[14] S. Kumar et al., Biophys. J. 82, 2360 (2002).

[15] J. C. Butler et al., Phys. Rev. Lett. 91, 028301 (2003).

[16] C. Storm et al., Nature (London) 435, 191 (2005).

[17] Y.-C. Lin et al., (unpublished).

[18] T. Odijk, Macromolecules 28, 7016 (1995).

[19] F. Gittes and F. C. MacKintosh, Phys. Rev. E 58, R1241 (1998).

[20] Z. Dogic et al., Phys. Rev. Lett. 92, 125503 (2004).

[21] N. Mucke et al., J. Mol. Biol. 335, 1241 (2004).

[22] P. A. Janmey et al., J. Cell Biol. 113, 155 (1991).

[23] M. Schopferer et al., J. Mol. Biol. 388, 133 (2009).

[24] R. Tharmann et al., Phys. Rev. Lett. 98, 088103 (2007).

[25] S. Rammensee et al., Eur. Biophys. J. 36, 661 (2007).

[26] S. Koester (private communication). 